\title{
Penile microvascular endothelial function in hypertensive patients: effects of acute type 5 phosphodiesterase inhibition
}

\author{
V. Verri ${ }^{1}$, A.A. Brandão ${ }^{3}$ and E. Tibirica ${ }^{1,2}$ \\ ${ }^{1}$ Instituto Nacional de Cardiologia, Ministério da Saúde, Rio de Janeiro, Brasi \\ ${ }^{2}$ Laboratório de Investigação Cardiovascular, Instituto Oswaldo Cruz, Rio de Janeiro, Brasi \\ ${ }^{3}$ Universidade do Estado do Rio de Janeiro, Rio de Janeiro, Brasil
}

\begin{abstract}
The primary aim of this study was to evaluate penile endothelial microvascular function in patients with primary arterial hypertension and age-matched normotensive subjects using laser speckle contrast imaging (LSCI). Additionally, we analyzed the acute penile microvascular effects induced by oral phosphodiesterase type 5 inhibitor (sildenafil; SIL) administration. Endothelium-dependent microvascular reactivity was evaluated in the penises and forearms of hypertensive patients (aged $58.8 \pm$ 6.6 years, $n=34)$ and age-matched healthy volunteers $(n=33)$ at rest and $60 \mathrm{~min}$ following oral SIL $(100 \mathrm{mg})$ administration. LSCI was coupled with cutaneous acetylcholine (ACh) iontophoresis using increasing anodal currents. Basal penile cutaneous vascular conductance (CVC) values were not significantly different between control subjects and hypertensive individuals. Penile CVC values increased significantly after SIL administration in control $(P<0.0001)$ and hypertensive $(P<0.0001)$ subjects. Peak CVC values were not different between the two groups during penile ACh iontophoresis before SIL administration ( $P=0.2052)$. Peak CVC values were higher in control subjects than in hypertensive subjects after SIL administration $(P=0.0427)$. Penile endothelium-dependent microvascular function is, to some extent, preserved in patients presenting with primary arterial hypertension under effective anti-hypertensive treatment. LSCI may be a valuable non-invasive tool for the evaluation of penile vascular responses to phosphodiesterase type 5 inhibitor.
\end{abstract}

Key words: Microvascular dysfunction; Hypertension; Laser speckle contrast imaging; Penile microcirculation

\section{Introduction}

Arterial hypertension is the result of complex interactions between environmental and genetic factors that initiate and perpetuate blood pressure elevations and is one of the most prevalent cardiovascular diseases worldwide, affecting approximately $25 \%$ of the global population. Arterial hypertension is characterized by significant systemic microvascular reactivity alterations, as demonstrated by studies reporting abnormal microvascular endothelial responses to a variety of stimuli in hypertensive patients, responses identified mostly via laser perfusion monitoring technology $(1,2)$. Moreover, several investigators have demonstrated reductions in capillary density and/or reactivity in experimental models of hypertension (3), patients with hypertension $(4,5)$, people with borderline hypertension (6), and normotensive subjects with a familial predisposition for developing hypertension (7). Given that microvascular dysfunction and rarefaction are unquestionably involved in arterial hypertension pathophysiology and that hypertension can lead to microvascular alterations, a bi-directional causality most likely exists between the two conditions.

In addition to having higher cardiovascular risks, hypertensive patients frequently have impaired quality of life because of vasculogenic erectile dysfunction (ED) (8). The prevalence of ED in hypertensive patients is significantly higher than that in age-matched normotensive subjects (8). It is now accepted that vasculogenic ED is associated with systemic vascular endothelial dysfunction and may be considered an early and independent marker of increased cardiovascular risk (9). Moreover, ED is associated with classical atherothrombotic disorder risk factors, such as age, sedentary lifestyle, hypertension, dysglycemia, dyslipidemia, obesity and smoking (10). It has also been suggested that patients with hypertension and ED who do not respond to type 5 phosphodiesterase enzyme (PDE-5) inhibitors may be at increased risk for cardiovascular events (11).

Correspondence: E. Tibiriçá: <etibi@ioc.fiocruz.br>

Received July 9, 2017 | Accepted September 25, 2017 
The pathophysiology of ED in hypertensive patients is characterized by vascular endothelial dysfunction caused by reduced nitric oxide (NO) bioavailability and increased oxidative stress resulting from increases in NADPH oxidase activity driven by angiotensin II that ultimately result in reduced corpus cavernosum blood vessel vasodilatory capacity (12). PDE-5 inhibitors have been shown to be effective and safe in patients with cardiovascular disease and to restore penile vasculature vasodilation (13). In patients with arterial hypertension, sildenafil improves ED without exerting any serious adverse effects on blood pressure (13). PDE-5 inhibitors reduce cyclic guanosine monophosphate (cGMP) catabolism, thereby increasing cGMP levels and enhancing vasodilation (14). Moreover, PDE-5 inhibitors significantly increase NO levels (15).

Laser speckle contrast imaging (LSCI) provides an innovative approach for non-invasive evaluation of skin microvascular endothelial function (16). A major advantage of this technique is that $\mathrm{LSCl}$ is more reproducible than earlier procedures, such as laser Doppler flowmetry and laser Doppler imaging (17). LSCI has also previously been shown to be an effective noninvasive technique for evaluating systemic microvascular reactivity in patients presenting with cardiovascular and metabolic diseases $(16,18)$. Moreover, we recently showed that $\mathrm{LSCl}$ is a valuable non-invasive method for evaluating penile microvascular endothelial function in healthy volunteers (19).

The primary objective of this study was to evaluate penile endothelial microvascular function in hypertensive patients and age-matched normotensive subject using LSCI. The secondary objective of this study was to determine the acute effects of oral sildenafil $(100 \mathrm{mg})$ administration on penile and systemic microvascular endothelial function.

\section{Material and Methods}

\section{Study design}

The present study was undertaken in accordance with the Helsinki Declaration of 1975, revised in 2000, and was approved by the Institutional Review Board (IRB) of the National Institute of Cardiology, Rio de Janeiro, RJ, Brazil, under protocol \#CAAE17663813.4.0000.5272 and registered at ClinicalTrials.gov (NCT02620995). Once deemed eligible to participate in this study, all subjects read and signed an informed consent document approved by the IRB.

Thirty-four hypertensive male outpatients who were eligible for this study were recruited from our hypertension clinics and from referrals by colleagues. The clinical characteristics of these patients are presented in Tables 1 and 2 . All subjects underwent complete medical examinations, which included searches for target organ lesions. Ambulatory hypertension was diagnosed using a clinically validated Omron Intellisense M7 upper arm blood pressure monitor (Omron Healthcare Europe B.V., Hoofddorp, The Netherlands) as follows: each subject rested for $10 \mathrm{~min}$ in the supine position in a cool $\left(23 \pm 1^{\circ} \mathrm{C}\right)$ and quiet environment before undergoing blood pressure measurements in both arms. The arm exhibiting the higher blood pressure was used for subsequent evaluations. Each patient then underwent measurements in the upright (orthostatic) position and three measurements in the sitting position at 1 -min intervals. The mean values of the two last measurements were used in this study. Insulin resistance was measured using the homeostatic model assessment (HOMA-IR) and calculated as the product of fasting plasma insulin (in $\mu \mathrm{Ul} / \mathrm{mL}$ ) multiplied by 0.0555 and as the quotient of fasting plasma glucose (in $\mathrm{mg} / \mathrm{dL}$ ) divided by 22.5 .

Sexually active male hypertensive patients aged 50 to 70 years with blood pressure levels $<160 / 100 \mathrm{mmHg}$ while receiving anti-hypertensive pharmacological treatment were included in this study. All patients with hypertension were evaluated via 24-h ambulatory blood pressure monitoring using oscillometric devices (Spacelabs, USA). The monitor was installed on the non-dominant arm between 7:00 and 9:00 am and removed $24 \mathrm{~h}$ later. Recordings were made every $15 \mathrm{~min}$ from 7:00 am to 11:00 pm (diurnal BP values) and every $30 \mathrm{~min}$ from 11:00 pm to 7:00 am (nocturnal BP values).

Patients with a history of diabetes mellitus, coronary artery disease, secondary arterial hypertension, neurologic or psychiatric disorders, or decompensated kidney or liver disease were excluded from this study. Patients using nitrates or alpha-adrenergic blockers were also excluded from this study. Patients should not have taken PDE-5 inhibitors within 30 days before enrolling in the study.

Thirty-three age-matched normotensive volunteers who were free of cardiovascular disease served as normotensive controls (Table 1). This group was considered a "reference" group for microvascular parameters. All patients and control subjects underwent detailed histories and physical examinations.

All evaluations were performed in the morning between 8:00 am and 12:00 pm after a 12-h fast. Blood specimens were collected, and subjects were asked to lie down for $20 \mathrm{~min}$ in a quiet environment with a constant temperature of $23 \pm 1^{\circ} \mathrm{C}$. All examinations followed the same sequence, beginning with $\mathrm{LSCl}$ microvascular reactivity measurements in the skin of the forearm, followed by the same measurements in the skin of the penis. The microvascular tests were performed before and $60 \mathrm{~min}$ after oral sildenafil $(100 \mathrm{mg})$ administration.

\section{Evaluation of microcirculatory reactivity}

The microcirculatory tests were performed in an undisturbed quiet room with a defined stable temperature $(23 \pm$ $1^{\circ} \mathrm{C}$ ) after a 20 -min rest period in the supine position. The room temperature was monitored and adjusted if necessary using air conditioning, as the outside temperature was usually $>25^{\circ} \mathrm{C}$. Iontophoresis was performed in the forearm $60 \mathrm{~min}$ after oral sildenafil administration and in the penis approximately $15 \mathrm{~min}$ after the forearm procedure 
Table 1. Characteristics of healthy volunteers and hypertensive patients.

\begin{tabular}{|c|c|c|c|}
\hline Parameters & Healthy volunteers $(n=33)$ & Hypertensive patients $(n=34)$ & $P$ value \\
\hline Age (years) & $56.9 \pm 5.0$ & $58.8 \pm 6.6$ & 0.2102 \\
\hline Body weight $(\mathrm{kg})$ & $77.7 \pm 11.9$ & $84.6 \pm 13.0$ & 0.0260 \\
\hline Body mass index $\left(\mathrm{kg} / \mathrm{m}^{2}\right)$ & $25.9 \pm 3.3$ & $29.6 \pm 4.4$ & 0.0004 \\
\hline Waist circumference $(\mathrm{cm})$ & $93.0 \pm 8.9$ & $102.0 \pm 9.3$ & 0.0002 \\
\hline Fasting glucose (mg/dL) & $99.4 \pm 7.3$ & $102.7 \pm 10.2$ & 0.1422 \\
\hline Glycated hemoglobin (\%) & $5.3 \pm 0.4$ & $5.6 \pm 0.3$ & 0.0211 \\
\hline Fasting insulin $(\mu \mathrm{Ul} / \mathrm{mL})$ & $7.2(5.5-10.0)$ & $12.4(7.4-15.9)$ & 0.0009 \\
\hline HOMA-IR index & $1.82(1.36-2.34)$ & $2.99(1.96-4.25)$ & 0.0007 \\
\hline Total cholesterol (mg/dL) & $217.4 \pm 26.4$ & $201.1 \pm 40.3$ & 0.0566 \\
\hline LDL cholesterol (mg/dL) & $142.9 \pm 25.8$ & $124.0 \pm 33.5$ & 0.0121 \\
\hline HDL cholesterol (mg/dL) & $44.9 \pm 8.1$ & $43.3 \pm 10.6$ & 0.5140 \\
\hline Triglycerides (mg/dL) & $141.0(95.0-182.0)$ & $155.0(101.8-212.8)$ & 0.2617 \\
\hline Urea $(\mathrm{mg} / \mathrm{dL})$ & $31.6 \pm 9.4$ & $33.1 \pm 9.3$ & 0.4934 \\
\hline Creatinine (mg/dL) & $0.9(0.8-1.0)$ & $0.9(0.8-1.1)$ & 0.6201 \\
\hline Uric acid $(\mathrm{mg} / \mathrm{dL})$ & $5.9 \pm 1.2$ & $6.5 \pm 1.5$ & 0.1001 \\
\hline Microalbumin (mg/L) & $5.0(1.0-7.4)$ & $5.0(1.5-10.0)$ & 0.4140 \\
\hline hs-CRP & $0.14(0.09-0.21)$ & $0.20(0.09-0.37)$ & 0.3593 \\
\hline Office SAP (mmHg) & $122.6 \pm 8.8$ & $136.7 \pm 13.8$ & $<0.0001$ \\
\hline Office DAP (mmHg) & $79.3 \pm 6.6$ & $86.7 \pm 7.6$ & $<0.0001$ \\
\hline Office MAP (mmHg) & $93.7 \pm 6.8$ & $103.4 \pm 8.7$ & $<0.0001$ \\
\hline \multicolumn{4}{|l|}{ ABPM } \\
\hline Mean 24-h SAP (mmHg) & - & $127.0(124.0-135.3)$ & \\
\hline Mean 24-h DAP (mmHg) & - & $82.5(75.7-86.5)$ & \\
\hline Mean daytime SAP $(\mathrm{mmHg})$ & - & $130.5(126.0-135.8)$ & \\
\hline Mean daytime DAP $(\mathrm{mmHg})$ & - & $85.0(77.7-88.0)$ & \\
\hline Mean nighttime SAP $(\mathrm{mmHg})$ & - & $116.0(112.0-128.3)$ & \\
\hline Mean nighttime DAP $(\mathrm{mmHg})$ & - & $73.2 \pm 9.7$ & \\
\hline \multicolumn{4}{|l|}{ MAP-F } \\
\hline PRE (mmHg) & $96.3 \pm 9.9$ & $108.0 \pm 8.6$ & $<0.0001$ \\
\hline POST (mmHg) & $90.3 \pm 8.4^{*}$ & $99.3 \pm 9.1^{*}$ & $<0.0001$ \\
\hline$\Delta \mathrm{MAP}-\mathrm{F}$ & $-6.0 \pm 7.2$ & $-8.8 \pm 6.1$ & 0.0980 \\
\hline
\end{tabular}

Results are reported as means \pm SD. Values that did not follow a Gaussian distribution are reported as medians (25th-75th percentile) (Shapiro-Wilk normality test). $\mathrm{P}$ values were estimated using two-tailed unpaired Student's $t$-tests or Mann-Whitney $U$ tests, as appropriate. $P$ values in bold type denote statistically significant differences. MAP values were obtained during a microcirculatory flowmetry (MAP-F) before (PRE) and after (POST) oral administration of a single dose of sildenafil (100 mg). $\Delta$ MAP-F indicates the reduction in MAP after sildenafil. ABPM: ambulatory blood pressure monitoring; DAP: diastolic arterial pressure; HOMA-IR: homeostatic model assessment of insulin resistance; hs-CRP: highsensitivity C-reactive protein; MAP, mean arterial pressure; SAP: systolic arterial pressure. ${ }^{*} \mathrm{P}<0.0001$, compared with MAP-F PRE.

because it was technically impossible to perform both procedures simultaneously.

\section{Evaluation of skin microvascular flow and reactivity}

Microvascular reactivity was evaluated using an LSCI system with a laser wavelength of $785 \mathrm{~nm}$ (PeriCam PSI system, Perimed, Sweden), which enabled us to perform noninvasive and continuous measurements of cutaneous microvascular perfusion changes, measured in arbitrary perfusion units (APU). The images were analyzed using the indicated software (PIMSoft, Perimed).
High-frame-rate $\mathrm{LSCl}$ is a recently marketed noncontact technique based on the analysis of the variations in speckle contrast, which allows measurements of fast changes in skin microvascular blood flow over wide skin areas with very good inter-day reproducibility (17). In fact, $\mathrm{LSCl}$ measures skin perfusion over wide areas (up to $100 \mathrm{~cm}^{2}$ ) with a high frequency (up to 100 images/s). LSCI thus presents the advantages of good temporal and spatial resolutions (20).

One skin site on the ventral surface of the forearm was randomly chosen for the recordings. Hair, broken skin, 
Table 2. Antihypertensive and cardiovascular drugs used by hypertensive patients.

\begin{tabular}{lc}
\hline Drugs & Hypertensive patients $(\mathrm{n}=34)$ \\
\hline Angiotensin II receptor blockers & $26(76.5)$ \\
Angiotensin converting enzyme inhibitors & $9(26.5)$ \\
Calcium channel blockers & $17(50)$ \\
$\beta$-adrenergic blockers & $5(14.7)$ \\
Diuretics & $24(70.6)$ \\
Statins & $9(26.5)$ \\
Fibrates & $2(5.9)$ \\
\hline
\end{tabular}

Data are reported as $\mathrm{n}(\%)$.

areas of skin pigmentation and visible veins were avoided, and two drug-delivery electrodes were installed using adhesive discs (LI 611, Perimed). The following two measurement areas were identified: a measurement area within the electrode $(\mathrm{ACh})$ and another measurement area (baseline control) adjacent to the electrode. A vacuum cushion (AB Germa, Sweden) was used to minimize recording artifacts generated by arm movements. ACh $2 \% \mathrm{w} / \mathrm{v}$ (Sigma Chemical Co., USA) iontophoresis was performed using a micropharmacology system (PF 751 Perilont USB Power Supply, Perimed, Sweden) with increasing anodal currents of 30,60, 90, 120, 150, and $180 \mu \mathrm{A}$, which were administered for 10 -s intervals spaced $1 \mathrm{~min}$ apart. The total charges for the above currents were $0.3,0.6,0.9,1.2,1.5$, and $1.8 \mathrm{mC}$, respectively. The dispersive electrode was attached approximately $15 \mathrm{~cm}$ from the electrophoresis chamber. The pharmacological test results are reported as peak values representing the maximal vasodilation observed after the highest Ach dose. Skin blood flow measurements in APU were divided by mean arterial pressure values to yield cutaneous vascular conductance (CVC) in APU/mmHg.

Finally, to evaluate penile skin microcirculation, the electrode was positioned on the base of the penis to perform iontophoretic ACh delivery, as described above and in a previous work (19). In this case, the dispersive electrode was attached to the thigh of each research subject. We used a fenestrated drape with a round opening in the center to lessen the effects of movement on the penile microvascular blood flow recordings. The penis was introduced through the opening of the drape and fixed to the drape using medical pressure-sensitive adhesive tape (Henkel Adhesive North America, USA). This tape features a hypoallergenic adhesive that is designed to hold firmly to the skin and dressing materials but can be easily removed without damaging the skin. The recordings of penile microvascular flow were always performed on a flaccid penis.

\section{Statistical analysis}

The results are reported as means $\pm S D$. Values that did not follow a Gaussian distribution are presented as medians (25th-75th percentile; Shapiro-Wilk normality test). The results were analyzed using two-tailed paired or unpaired Student's t-tests, Wilcoxon matched pairs tests, the Mann-Whitney U-test or repeated measures ANOVA followed by Dunnett's multiple comparison test when appropriate. $P$ values $<0.05$ were considered to be statistically significant. All statistical analyses were performed using Prism, version 6.0 (GraphPad Software Inc. USA).

\section{Results}

\section{Clinical characteristics of the study subjects}

The clinical characteristics of the healthy volunteers and hypertensive patients, including information regarding current cardiovascular drug use, are presented in Tables 1 and 2. Anthropometric parameters (body weight, body mass index and waist circumference) were significantly higher in hypertensive patients than in normotensive controls.

Systolic, diastolic and mean arterial blood pressure values were significantly higher in hypertensive patients than in normotensive controls. Moreover, mean arterial pressure measured during microvascular flowmetry (MAP-F) was higher in hypertensive patients than in normotensive controls, both before and after sildenafil administration. We also observed a significant decrease in mean arterial pressure after sildenafil administration in both study groups. However, there was no significant difference in the size of this decrease between the two groups (Table 1).

LDL-cholesterol plasma levels were lower in hypertensive patients than in normotensive controls, likely because $26.5 \%$ of patients were taking lipid-lowering drugs (statins). No other clinical parameter was different between patients and controls.

\section{Evaluation of skin microvascular flow and reactivity}

Penile skin ACh iontophoresis induced significant, current-related increases in microvascular CVC (before and after SIL administration), and CVC was significantly higher after SIL administration than before SIL administration in both healthy controls and hypertensive subjects (Figure 1). 

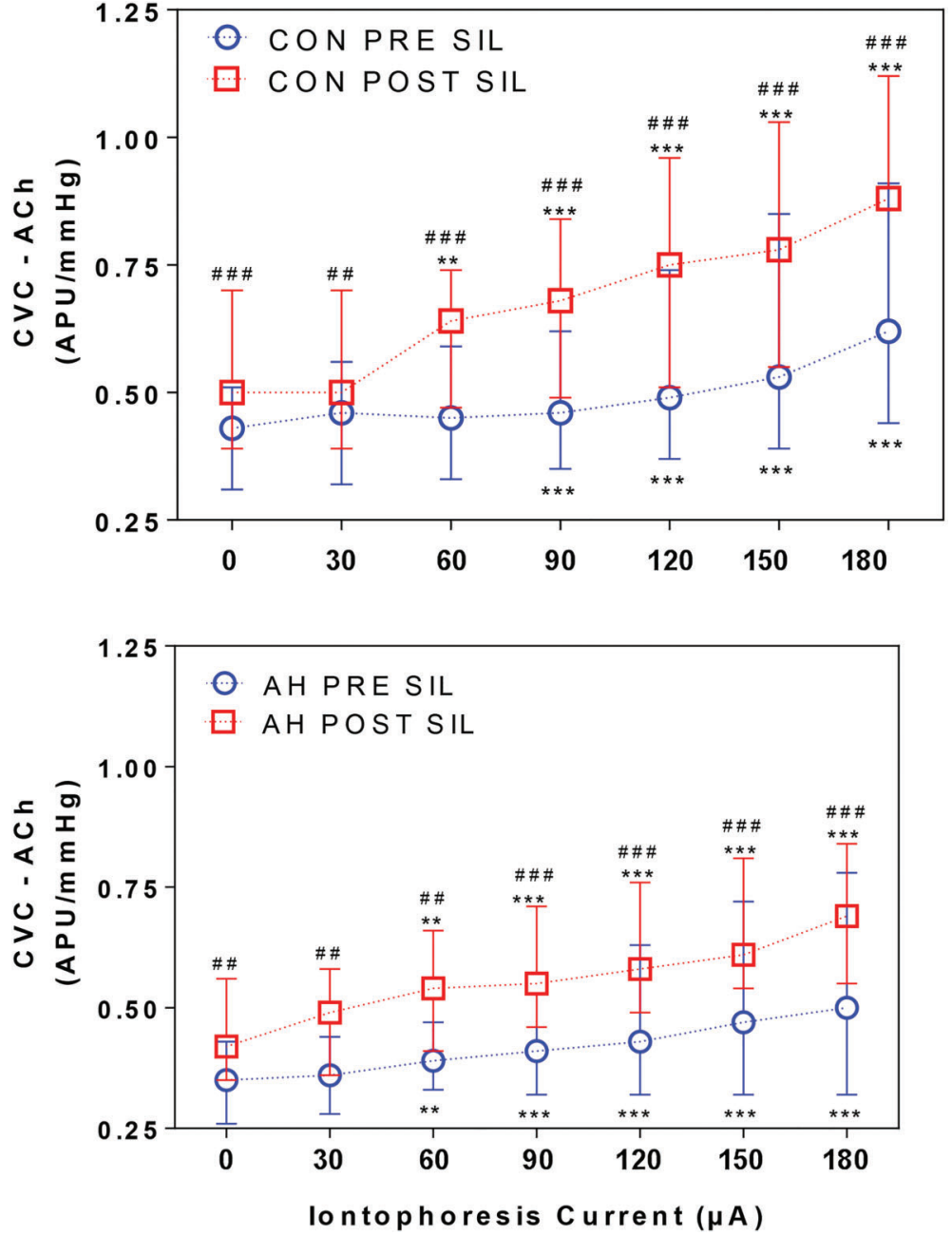

Figure 1. Effects of penile skin acetylcholine (ACh) iontophoresis on cutaneous microvascular conductance (CVC), in arbitrary perfusion units (APU) divided by mean arterial pressure in $\mathrm{mmHg}$ in healthy volunteers (upper panel, $\mathrm{CON}$; $n=33$ ) and hypertensive patients (lower panel, $\mathrm{AH}$; $n=34$ ) before (PRE SIL) and after (POST SIL) oral sildenafil $(100 \mathrm{mg})$ administration. Data are reported as medians (interquartile range) and were analyzed using Wilcoxon's test, the MannWhitney U-test or repeated measures analysis of variance followed by Dunnett's multiple comparison test when appropriate. ${ }^{* *} \mathrm{P}<0.01$ and ${ }^{* * *} \mathrm{P}<0.001$ compared with baseline values. ${ }^{\# \#} \mathrm{P}<0.01$ and ${ }^{\# \# \#} \mathrm{P}<0.001$ compared with PRE SIL.
Before SIL administration, the basal penile skin microvascular flow values were 37.0 (28.5-48.5) and 38.0 (27.0-46.2) APU in control and hypertensive participants, respectively $(P=0.5808)$. After $S I L$ administration, the flow values were $46.0(39.0-57.5)$ and 42.5 (31.5-54.7) APU in control and hypertensive participants, respectively $(P=0.4256)$. Penile baseline microvascular flow was significantly increased after SIL administration compared with before SIL administration in control $(P=0.0006)$ and hypertensive $(P=0.0038)$ subjects. Before SIL administration, the basal penile CVC values were $0.43(0.31-0.51)$ and 0.35 (0.26-0.43) APU/mmHg in control and hypertensive participants, respectively $(\mathrm{P}=0.0590)$. After SIL administration, the flow values were $0.50(0.39-0.70)$ and $0.42(0.35-0.56)$ $\mathrm{APU} / \mathrm{mmHg}$ in control and hypertensive participants, respectively ( $P=0.0857)$. Penile baseline $C V C$ values increased significantly after SIL administration compared with before SIL administration (Figure 1$)$ in control $(P<0.0001)$ and hypertensive $(P<0.0001)$ subjects.
Before SIL administration, the peak CVC values during penile ACh iontophoresis were $0.62(0.44-0.91)$ and 0.50 (0.33-0.79) APU/mmHg in control and hypertensive participants, respectively $(P=0.2052)$. After $\mathrm{SIL}$ administration, the peak $\mathrm{CVC}$ values during penile $\mathrm{ACh}$ iontophoresis were $0.88(0.66-1.12)$ and $0.69(0.56-0.84) \mathrm{APU} / \mathrm{mmHg}$ in control and hypertensive participants, respectively $(\mathrm{P}=$ 0.0427 ). In control subjects, the peak penile CVC values resulting from $\mathrm{ACh}$ iontophoresis were significantly higher after SIL administration than before SIL administration $(P<0.0001)$; the same was true in hypertensive subjects $(P=0.0006$; Figure 1).

There were no significant correlations between mean arterial pressure changes following sildenafil administration and penile microvascular reactivity (Table 3 ).

A subgroup analyses showed that maximum endothelium-dependent penile vasodilation was reduced in the group of patients treated with $\beta$-blockers $(n=5)$, compared with the whole group of patients. On the other hand, penile 
vasodilation in the subgroup of patients treated with statins was comparable to that observed in the whole group of patients (Table 4).

\section{Systemic microvascular reactivity}

Forearm skin ACh iontophoresis also induced significant, current-related increases in microvascular CVC (before and after SIL administration); however, CVC was higher only in hypertensive subjects following SIL administration (Figure 2).

Before SIL administration, the basal forearm skin microvascular flow values were 25.0 (22.0-28.5) and 25.5 (21.5-28.0) APU in control and hypertensive participants, respectively $(P=0.6556)$. After $\mathrm{SIL}$, the flow values were 22.0 (20.0-27.0) and 25.0 (18.7-29.0) APU in control and hypertensive participants, respectively $(P=0.4177)$. Forearm baseline microvascular flow was not significantly increased after SIL administration compared with before SIL administration in control $(P=0.2837)$ or hypertensive

Table 3. Correlations between mean arterial pressure changes induced by sildenafil, and endothelium-dependent systemic and penile microvascular reactivity in hypertensive patients.

\begin{tabular}{lc}
\hline Parameter & $\Delta$ MAP $(\mathrm{mmHg}) / r(\mathrm{P}$ value $)$ \\
\hline CVC max (APU/mmHg) & \\
Systemic & $-0.268(0.125)$ \\
Penile & $-0.268(0.125)$ \\
$\Delta$ CVC (APU/mmHg) & \\
Systemic & $-0.254(0.1480)$ \\
Penile & $-0.199(0.2580)$ \\
\hline
\end{tabular}

r. Spearman's rank correlation coefficient; CVC max: maximum effects of skin acetylcholine iontophoresis on cutaneous microvascular conductance (CVC) in arbitrary perfusion units of flow (APU) divided by mean arterial pressure in $\mathrm{mmHg} ; \triangle \mathrm{CVC}$ : increases in CVC induced by skin acetylcholine iontophoresis compared to baseline values; $\triangle$ MAP: decreases in mean arterial pressure induced by sildenafil.
$(P=0.8222)$ subjects. Before SIL administration, the basal CVC values were $0.27(0.21-0.30)$ and $0.23(0.19-0.0 .26)$ $\mathrm{APU} / \mathrm{mmHg}$ in control and hypertensive participants, respectively ( $P=0.0274)$. After SIL administration, the CVC values were $0.24(0.21-0.30)$ and $0.24(0.19-0.29)$ APU/ $\mathrm{mmHg}$ in control and hypertensive participants, respectively $(P=1.0)$. Forearm baseline CVC did not change significantly after SIL administration compared with before SIL administration in control $(P=0.5494)$ or hypertensive $(P=0.0671)$ subjects (Figure 2).

Before SIL administration, the peak CVC values during forearm ACh iontophoresis were $0.58(0.42-0.73)$ and $0.45(0.32-0.70)$ APU $/ \mathrm{mmHg}$ in control and hypertensive participants, respectively $(P=0.1083)$. After SIL administration, the peak CVC values during forearm ACh iontophoresis were $0.59(0.51-0.74)$ and $0.50(0.37-0.67)$ $\mathrm{APU} / \mathrm{mmHg}$ in control and hypertensive participants, respectively $(P=0.0378)$. In control subjects, the peak forearm CVC values resulting from ACh iontophoresis after SIL administration were not significantly different from those resulting from ACh iontophoresis before SIL administration $(P<0.3545)$; however, in hypertensive subjects, there was an increase in peak CVC after SIL administration compared with before SIL administration $(P=0.0497$; Figure 1).

There were no significant correlations between mean arterial pressure changes following SIL administration and systemic (forearm) microvascular reactivity (Table 3).

\section{Discussion}

The main findings of this study are as follows: i) overall endothelial-dependent penile microvascular vasodilation decreased in hypertensive patients compared with normotensive age-matched control subjects; ii) acute SIL administration significantly increased penile microvascular vasodilation in both normotensive and hypertensive individuals; iii) control subjects experienced greater maximum increases in penile microvascular conductance induced

Table 4. Effects of penile acetylcholine iontophoresis on endothelium-dependent cutaneous microvascular conductance in hypertensive patients before (PRE SIL) and after (POST SIL) oral sildenafil $(100 \mathrm{mg})$ administration, according to pharmacological treatments.

\begin{tabular}{lllc}
\hline & \multicolumn{3}{c}{ CVC $\max ($ APU $/ \mathrm{mmHg})$} \\
\cline { 2 - 4 } & All patients $(\mathrm{n}=34)$ & Beta-blockers $(\mathrm{n}=5)$ & Statins $(\mathrm{n}=9)$ \\
\hline PRE SIL & $0.50(0.32-0.79)$ & $0.32(0.26-0.46)^{\#}$ & $0.58(0.42-0.68)$ \\
POST SIL & $0.69(0.56-0.84)^{* * *}$ & $0.44(0.31-0.54)^{\# \#}$ & $0.57(0.43-0.72)$ \\
\hline
\end{tabular}

Data are reported as medians (interquartile range). CVC max: maximum effects of skin acetylcholine iontophoresis on cutaneous microvascular conductance in arbitrary perfusion units of flow (APU) divided by mean arterial pressure in $\mathrm{mmHg}$. ${ }^{* * *} P<0.001$ compared with PRE SIL. ${ }^{\#} \mathrm{P}<0.05$ and ${ }^{\# \#} \mathrm{P}<0.01$ compared with all patients (Mann-Whitney U-test). 

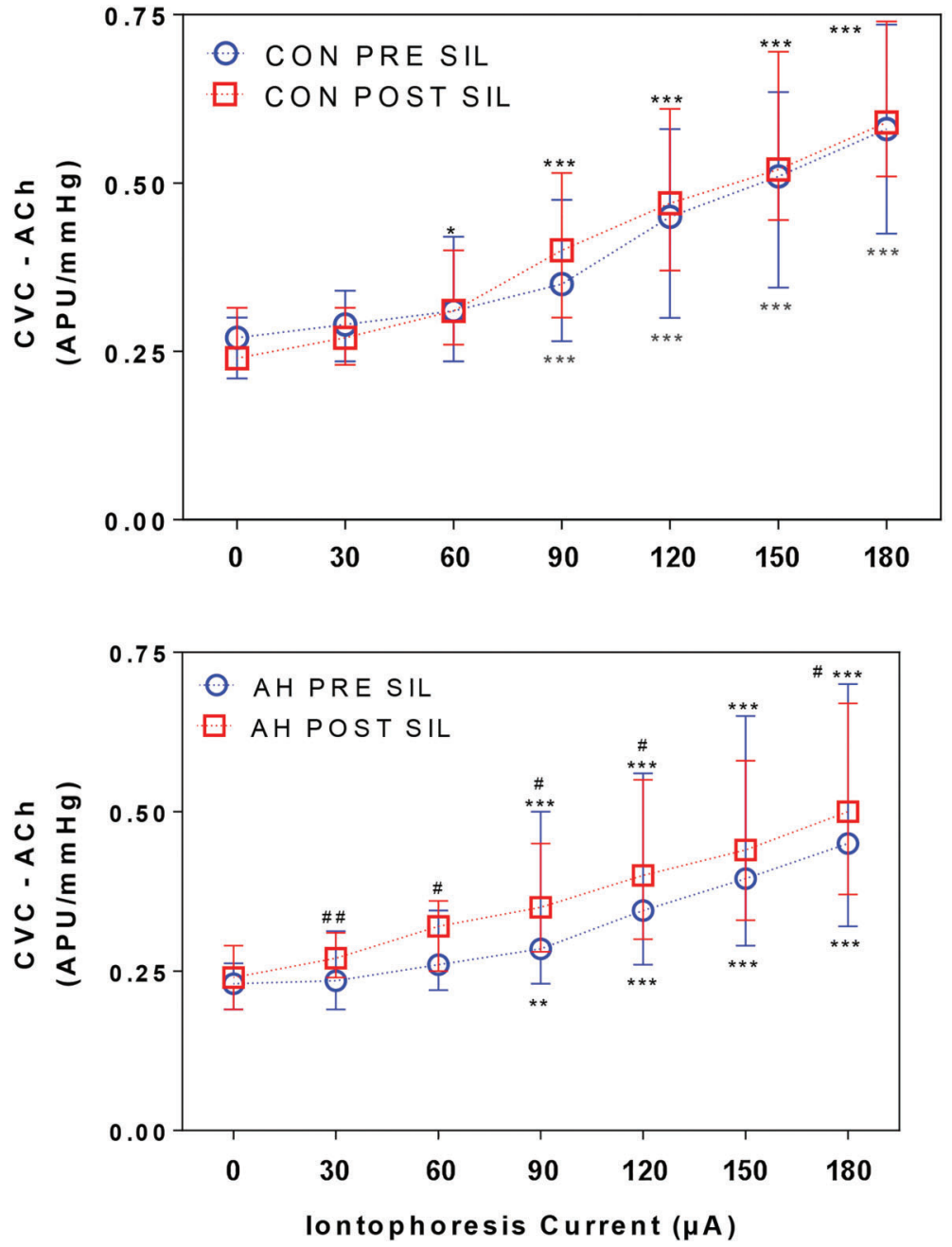

Figure 2. Effects of forearm skin acetylcholine (ACh) iontophoresis on cutaneous microvascular conductance (CVC), in arbitrary perfusion units (APU) divided by mean arterial pressure in $\mathrm{mmHg}$ in healthy volunteers (upper panel, $\mathrm{CON} ; \mathrm{n}=33$ ) and hypertensive patients (lower panel, $\mathrm{AH}$; $n=34)$ before (PRE SIL) and after (POST SIL) oral sildenafil (100 $\mathrm{mg})$ administration. Data are reported as medians (interquartile range) and were analyzed using Wilcoxon's test, the MannWhitney U-test or repeated measures analysis of variance followed by Dunnett's multiple comparison test when appropriate. ${ }^{*} \mathrm{P}<0.05,{ }^{* *} \mathrm{P}<0.01$ and ${ }^{* * *} \mathrm{P}<0.001$ compared with baseline values. ${ }^{\#} \mathrm{P}<0.05$ and ${ }^{\# \#} \mathrm{P}<0.01$ compared with PRE SIL. by acetylcholine iontophoresis after SIL administration than hypertensive patients, and iv) SIL increased systemic endothelial-dependent microvascular vasodilation in hypertensive patients, but not normotensive subjects.

In our study, hypertensive patients exhibited greater mean body weight, body mass index and waist circumference than age-matched normotensive individuals. These findings support those of previous studies demonstrating the existence of well-known links between different components of metabolic syndrome, including overweight/ obesity and increased visceral fat deposition, and arterial hypertension (21). Moreover, maximum penile microvascular vasodilation was greater in normotensive individuals than in hypertensive patients after sildenafil administration. Although hypertensive patients also exhibited significantly increased microvascular responses to sildenafil, the global endothelium-dependent penile microvascular vasodilatory response induced by cutaneous acetylcholine iontophoresis appeared to be greater in normotensive controls than in hypertensive patients, both before and after sildenafil administration.

It is also important to mention that in hypertensive patients, ED may be a complication of antihypertensive treatment (22). Antihypertensive drugs that block the sympathetic nervous system, including central and peripheral sympatholytics and $\beta$-blockers, have been frequently linked to ED $(23,24)$. Several studies suggest that using diuretics and $\beta$-blockers other than nebivolol (25) commonly results in ED in hypertensive men $(26,27)$, while using antihypertensive agents that block the renin-angiotensin system, such as angiotensin-converting-enzyme inhibitors (ACE-i) and angiotensin II receptor blockers (ARBs), improves endothelial function and, consequently, erectile function in hypertensive patients $(22,23)$. Calcium-channel blockers (CCB) have been reported to have neutral effects on erectile function (28). Most hypertensive patients enrolled in the present study were receiving antihypertensive drugs that theoretically do not interfere with erectile function 
(CCBs, $50 \%$ of patients) or improve erectile function (ARBs or ACE-is, $100 \%$ of patients). Only a small proportion of patients $(\sim 15 \%)$ were receiving $\beta$-blockers. Most patients $(\sim 70 \%)$ were being treated with diuretics for medical reasons. In the present study, subgroup analyses showed that maximum endothelium-dependent penile microvascular vasodilation was significantly reduced compared to that observed in the whole group of patients. In contrast, in the subgroup of patients treated with statins microvascular vasodilation was comparable to that observed in the whole group of patients. Nevertheless, it is important to note that there were only 5 patients treated with $\beta$-blockers and nine patients treated with statins in our study; thus, the small sample size, and consequently reduced statistical power, could have biased statistical analysis of subgroups of patients.

Hypertensive patients included in the present study were under chronic and effective anti-hypertensive treatment, thus explaining the significant increase in baseline penile microvascular flow, as well as the substantial vasodilator response of the penile microcirculation to the stimulation with endothelial-dependent vasodilators. In fact, it is well documented that anti-hypertensive drugs that block the renin-angiotensin system induce significant improvements in macro- and microvascular endothelial function (29). In conclusion, the present study shows that penile microvascular reactivity is satisfactory in treated hypertensive patients.

Sildenafil-induced inhibition of PDE-5, an enzyme considered very specific for the vascular smooth muscle of the penis (10), is known to increase NO bioavailability in the penis and its supplying vasculature, resulting in vasodilation and increased blood flow (13). On the other hand, long-term treatment of patients with arterial hypertension with SIL (50 mg 3 times daily) induces significant reductions in systolic and diastolic ambulatory blood pressure (30). In patients with congestive heart failure, SIL treatment improves oxygen uptake, pulmonary vascular resistance, cardiac output, and exercise capacity (31). Consequently, PDE-5 inhibitors have been proposed for the treatment of several cardiovascular diseases $(15,31)$.

In contrast to the penile microcirculation, systemic microvascular endothelium-dependent vasodilation was

\section{References}

1. Kaiser SE, Sanjuliani AF, Estato V, Gomes MB, Tibirica E. Antihypertensive treatment improves microvascular rarefaction and reactivity in low-risk hypertensive individuals. Microcirculation 2013; 20: 703-716, doi: 10.1111/micc.12067.

2. Farkas K, Kolossvary E, Jarai Z, Nemcsik J, Farsang C. Non-invasive assessment of microvascular endothelial function by laser doppler flowmetry in patients with essential hypertension. Atherosclerosis 2004; 173: 97-102, doi: 10.1016/j.atherosclerosis.2003.11.015. not affected by acute SIL administration in normotensive subjects. However, in hypertensive patients, acute SIL administration induced significant increases in systemic microvascular vasodilation, although reduction in arterial pressure was not correlated to microvascular vasodilation increases. These results may be explained by the fact that NO bioavailability is significantly reduced in arterial hypertension, whereas oxidative stress is increased, leading to macrovascular and predominantly microvascular endothelial dysfunction, which is a hallmark of primary arterial hypertension pathophysiology $(32,33)$. Thus, acute increases in systemic NO bioavailability induced by SIL may enhance NO-mediated microvascular vasodilation in hypertensive patients, but not normotensive individuals. We and other groups have previously reported on functional and structural microvascular rarefaction, as well as reduced capillary recruitment, in spontaneously hypertensive rats $(34,35)$ and hypertensive patients $(1,5,36)$. Thus, it is reasonable to assume that although mean arterial blood pressure decreased in both experimental groups, and the reductions were not different between the groups, acute SIL administration improved systemic microvascular function in hypertensive patients, but not in normotensive individuals, via endothelium-dependent recruitment of previously closed microvessels. These findings suggest that systemic microvasculature responds differently to acute SIL administration than penile vasculature.

$\mathrm{LSCl}$ may well be a valuable non-invasive tool for the evaluation of penile microvascular responses to PDE-5 inhibitors in patients presenting with cardiovascular diseases.

\section{Acknowledgments}

Research supported by CNPq (Conselho Nacional de Desenvolvimento Científico e Tecnológico), FAPERJ (Fundação de Amparo à Pesquisa do Estado do Rio de Janeiro), and the Oswaldo Cruz Foundation (FIOCRUZ), Rio de Janeiro, Brazil. Part of the present work was presented during the 26th European Meeting on Hypertension and Cardiovascular Protection, Paris, France, June 2016.
3. Feihl F, Liaudet L, Waeber B, Levy B. Hypertension: a disease of the microcirculation? Hypertension 2006; 48: 1012-1017, doi: 10.1161/01.HYP.0000249510.20326.72.

4. Antonios TF, Rattray FE, Singer DR, Markandu ND, Mortimer PS, Macgregor GA. Maximization of skin capillaries during intravital video-microscopy in essential hypertension: comparison between venous congestion, reactive hyperaemia and core heat load tests. Clin Sci 1999; 97: 523-528, doi: 10.1042/cs0970523. 
5. Serne EH, Gans RO, Ter Maaten JC, Tangelder GJ, Donker AJ, Stehouwer CD. Impaired skin capillary recruitment in essential hypertension is caused by both functional and structural capillary rarefaction. Hypertension 2001; 38: 238242, doi: 10.1161/01.HYP.38.2.238.

6. Antonios TF, Singer DR, Markandu ND, Mortimer PS, Macgregor GA. Rarefaction of skin capillaries in borderline essential hypertension suggests an early structural abnormality. Hypertension 1999; 34: 655-658, doi: 10.1161/01.HYP. 34.4.655.

7. Antonios TF, Rattray FM, Singer DR, Markandu ND, Mortimer PS, Macgregor GA. Rarefaction of skin capillaries in normotensive offspring of individuals with essential hypertension. Heart 2003; 89: 175-178, doi: 10.1136/ heart.89.2.175.

8. Manolis A, Doumas M. Sexual dysfunction: the 'prima ballerina' of hypertension-related quality-of-life complications. J Hypertens 2008; 26: 2074-2084, doi: 10.1097/ HJH.0b013e32830dd0c6.

9. Shin D, Pregenzer G Jr, Gardin JM. Erectile dysfunction: a disease marker for cardiovascular disease. Cardiol Rev 2011; 19: 5-11, doi: 10.1097/CRD.0b013e3181fb7eb8.

10. Vlachopoulos CV, Terentes-Printzios DG, loakeimidis NK, Aznaouridis KA, Stefanadis $\mathrm{Cl}$. Prediction of cardiovascular events and all-cause mortality with erectile dysfunction: a systematic review and meta-analysis of cohort studies. Circ Cardiovasc Qual Outcomes 2013; 6: 99-109, doi: 10.1161/ CIRCOUTCOMES.112.966903.

11. Javaroni V, Queiroz-Miguez M, Abreu-Casanova M, Oigman W, Neves MF. Brachial flow-mediated dilation correlates with vardenafil response in hypertensive men with vasculogenic erectile dysfunction. Urology 2011; 78: 368-374, doi: 10.1016/j.urology.2011.02.070.

12. Gratzke C, Angulo J, Chitaley K, Dai YT, Kim NN, Paick JS, et al. Anatomy, physiology, and pathophysiology of erectile dysfunction. J Sex Med 2010; 7: 445-475, doi: 10.1111/ j.1743-6109.2009.01624.x.

13. Chrysant SG. Effectiveness and safety of phosphodiesterase 5 inhibitors in patients with cardiovascular disease and hypertension. Curr Hypertens Rep 2013; 15: 475-483, doi: 10.1007/s11906-013-0377-9.

14. Schwartz BG, Jackson G, Stecher VJ, Campoli-Richards DM, Kloner RA. Phosphodiesterase type 5 inhibitors improve endothelial function and may benefit cardiovascular conditions. Am J Med 2013; 126: 192-199, doi: 10.1016/j.amjmed.2012. 08.015 .

15. Nguyen $H$, Amanullah AM. Therapeutic Potentials of phosphodiesterase-5 inhibitors in cardiovascular disease. Rev Cardiovasc Med 2014; 15: 158-167.

16. Cordovil I, Huguenin G, Rosa G, Bello A, Kohler O, De Moraes R, et al. Evaluation of systemic microvascular endothelial function using laser speckle contrast imaging. Microvasc Res 2012; 83: 376-379, doi: 10.1016/j.mvr.2012. 01.004.

17. Roustit M, Millet C, Blaise S, Dufournet B, Cracowski Jl. Excellent reproducibility of laser speckle contrast imaging to assess skin microvascular reactivity. Microvasc Res 2010; 80: 505-511, doi: 10.1016/j.mvr.2010.05.012.

18. Souza EG, De Lorenzo A, Huguenin G, Oliveira GM, Tibirica E. Impairment of systemic microvascular endothelial and smooth muscle function in individuals with early-onset coronary artery disease: studies with laser speckle contrast imaging. Coron Artery Dis 2014; 25: 23-28, doi: 10.1097/MCA.0000000000000055.

19. Verri V, Brandao A, Tibirica $E$. The evaluation of penile microvascular endothelial function using laser speckle contrast imaging in healthy volunteers. Microvasc Res 2015; 99: 96-101, doi: 10.1016/j.mvr.2015.03.006.

20. Roustit M, Cracowski Jl. Assessment of endothelial and neurovascular function in human skin microcirculation. Trends Pharmacol Sci 2013; 34: 373-384, doi: 10.1016/j.tips.2013. 05.007.

21. Sperling LS, Mechanick JI, Neeland IJ, Herrick CJ, Despres JP, Ndumele CE, et al. The cardiometabolic health alliance: working toward a new care model for the metabolic syndrome. J Am Coll Cardiol 2015; 66: 1050-1067, doi: 10.1016/ j.jacc.2015.06.1328.

22. Javaroni V, Neves MF. Erectile dysfunction and hypertension: impact on cardiovascular risk and treatment. Int J Hypertens 2012; 2012: 627278, doi: 10.1155/2012/ 627278.

23. Fogari R, Preti P, Derosa G, Marasi G, Zoppi A, Rinaldi A, et al. Effect of antihypertensive treatment with valsartan or atenolol on sexual activity and plasma testosterone in hypertensive men. Eur J Clin Pharmacol 2002; 58: 177-180, doi: 10.1007/s00228-002-0456-3.

24. Fogari R, Zoppi A, Poletti L, Marasi G, Mugellini A, Corradi $\mathrm{L}$. Sexual activity in hypertensive men treated with valsartan or carvedilol: a crossover study. Am J Hypertens 2001; 14: 27-31, doi: 10.1016/S0895-7061(00)01214-0.

25. Brixius K, Middeke M, Lichtenthal A, Jahn E, Schwinger RH. Nitric oxide, erectile dysfunction and beta-blocker treatment (Mr Noed study): benefit of nebivolol versus metoprolol in hypertensive men. Clin Exp Pharmacol Physiol 2007; 34: 327-331, doi: 10.1111/j.1440-1681.2007.04551.x.

26. Wassertheil-Smoller S, Blaufox MD, Oberman A, Davis BR, Swencionis C, Knerr MO, et al. Effect of antihypertensives on sexual function and quality of life: the Taim study. Ann Intern Med 1991; 114: 613-620, doi: 10.7326/0003-4819114-8-613.

27. Viigimaa M, Doumas M, Vlachopoulos $C$, Anyfanti $P$, Wolf $J$, Narkiewicz K, et al. Hypertension and sexual dysfunction: time to act. J Hypertens 2011; 29: 403-407, doi: 10.1097/ HJH.0b013e328342c659.

28. Baumhakel M, Schlimmer N, Kratz M, Hackett G, Jackson G, Bohm M. Cardiovascular risk, drugs and erectile function a systematic analysis. Int J Clin Pract 2011; 65: 289-298, doi: 10.1111/j.1742-1241.2010.02563.x.

29. Ghiadoni L, Taddei S, Virdis A. Hypertension and endothelial dysfunction: therapeutic approach. Curr Vasc Pharmacol 2012; 10: 42-60, doi: 10.2174/157016112798829823.

30. Oliver JJ, Melville VP, Webb DJ. Effect of regular phosphodiesterase type 5 inhibition in hypertension. Hypertension 2006; 48: 622-627, doi: 10.1161/01.HYP.0000239816. 13007.c9.

31. Schwartz BG, Levine LA, Comstock G, Stecher VJ, Kloner RA. Cardiac uses of phosphodiesterase- 5 inhibitors. J Am Coll Cardiol 2012; 59: 9-15, doi: 10.1016/j.jacc.2011. 07.051.

32. Yannoutsos A, Levy BI, Safar ME, Slama G, Blacher J. Pathophysiology of hypertension: interactions between macro and microvascular alterations through endothelial dysfunction. 
J Hypertens 2014; 32: 216-224, doi: 10.1097/HJH.000000 0000000021.

33. Virdis A, Neves MF, Duranti E, Bernini G, Taddei S. Microvascular endothelial dysfunction in obesity and hypertension. Curr Pharm Des 2013; 19: 2382-2389, doi: 10.2174/ 1381612811319130006.

34. Sabino B, Lessa MA, Nascimento AR, Rodrigues CA, Henriques MG, Garzoni LR, et al. Effects of antihypertensive drugs on capillary rarefaction in spontaneously hypertensive rats: intravital microscopy and histologic analysis. J Cardiovasc
Pharmacol 2008; 51: 402-409, doi: 10.1097/FJC.0b013 e3181673bc5.

35. Nascimento AR, Lessa MA, Sabino B, Bousquet P, Tibirica E. Microvascular effects of centrally acting antihypertensive drugs in spontaneously hypertensive rats. J Cardiovasc Pharmacol 2010; 55: 240-247, doi: 10.1097/FJC.0b013e3181ce9810.

36. Debbabi H, Uzan L, Mourad JJ, Safar M, Levy BI, Tibirica E. Increased skin capillary density in treated essential hypertensive patients. Am J Hypertens 2006; 19: 477-483, doi: 10.1016/j.amjhyper.2005.10.021. 